\title{
Relação entre o Perfil do Usuário e a Escolha do Perfil do Tutor ${ }^{i}$
}

\author{
Adriana Justin Cerveira Kampff \\ Antônio da Fonseca de Lira** \\ Doris Simone Reitz*** \\ Fábio de Jesus Lima Gomes*** \\ Luís Carlos Costa Fonseca***** \\ Nádie Christina Ferreira Machado* \\ Magda Bercht ${ }^{* * * * * * *}$
}

\section{Resumo}

O presente trabalho apresenta um SSPT (Sistema de Sugestão de Perfis de Tutores). Os fundamentos do SSPT estão embasados nos conceitos de tipologia de personalidade de Carl Gustav Jung e nas escolhas sociométricas de Jacob Levy Moreno. Basicamente, o sistema se propõe a identificar e sugerir um tutor com perfil de personalidade similar ao do usuário, podendo este aceitar ou não a sugestão. A partir dessa decisão do usuário o sistema deverá gerar dados a serem armazenados em um banco de dados, para a validar ou refutar a hipótese que prevê a escolha de um agente com perfil semelhante ao do usuário, considerando as variáveis: gênero, raça, idade e área de conhecimento. Este trabalho pretende subsidiar a criação de um ambiente onde a aprendizagem seja potencializada através de uma interação baseada na aceitação mútua por parte do aprendiz e do tutor. Este último servindo como agente motivador no processo de aprendizagem, estimulando o processo cognitivo do aluno. Como trabalhos subjacentes, desenvolve-se uma pesquisa entre as relações de usuários alunos e tutores artificiais.

Palavras-chave: perfil, agentes, tipologia junguiana, sociometria.

\section{Relations between user profile and the choice of and tutor profile}

\section{Abstract}

The aim of this study is to present a SSPT - Sistema de Sugestão de Perfis de Tutores (Tutor Profile Suggestion System). As for SSPT fundaments we have used the typology personality concept of Carl Gustav Jung and, the sociometric choices of Jacob Levy Moreno. Basically, the system identifies and suggests a specific tutor (a kind of learning fellow agent) with a similar personality profile of the current user. The user is free to accept it or refuse the suggestion. After the user has taken his/her

\footnotetext{
* Graduada em Informática (PUC/RS), Mestre em Ciência da computação (UFRGS) e Doutoranda em Informática na Educação (UFRGS).

** Graduado em Licenciatura Plena em Matemática (FUA/AM), Mestre em Informática Industrial (CEFET/PR) e Doutorando em Informática na Educação (UFRGS). Bolsista FAPEAM.

*** Graduada em Educação Física (UFRGS), Mestre em Engenharia de Produção (UFRGS) e Doutoranda em Informática na Educação (UFRGS).

**** Graduado em Ciência da Computação (UESPI), Mestre em Ciência da Computação (UFPE) e Doutorando em Informática na Educação (UFRGS).

***** Graduado em Tecnologia em Processamento de Dados (UNICEUMA), Mestre em Engenharia Elétrica (UFMA) e Doutorando em Informática na Educação (UFRGS). Bolsista FAPEMA.

****** Graduada em Psicologia (UCPel), Mestre em Educação (UFRGS) e Doutoranda em Informática na Educação (UFRGS).

******* Doutora em Ciência da Computação (UFRGS). Professora no PGIE/UFRGS na Disciplina - Construindo objeto de pesquisa em Computação Afetiva - 2005/1, orientadora do projeto.
} 
decision about the tutor, the system should generate data which will be saved in a data base, in order to validate or not the hypothesis that it's likely that a tutor with a similar personality of his/her user is chosen. Four variables have been considered such as: gender, race, age and knowledge area. The present work is part of a bigger project concerning the implementation of an environment, in which learning is potencialized by a closer interaction, based on mutual acceptance between user and agent. Other goals that are part of this study are: an analysis of the relations that occur between users and tutors according to the variables mentioned above.

Key words: profile, agents, jungian typology, sociometry.

\section{Introdução}

O estudo sobre padrões de perfis de usuários e suas preferências em relação a tutores reflete o interesse dos pesquisadores em identificar características comuns em grupos de usuários (perfil psicológico, gênero, faixa etária, raça e área de conhecimento) e suas preferências em relação à aparência e ao perfil psicológico de um tutor para a aprendizagem.

Diversos trabalhos abordam o uso de tutores, muitas vezes apresentados em forma de desenho, humano ou não, normalmente, com aparência definida por critérios sem uma base de investigação mais ampla. Como por exemplo: ADELE (enfermeira), STEVE (personagem 3D), Vincent (personagem 2D), Cosmo (robô humanóide), Herman (inseto) e PAT (personagem 2D). O que se está pretendendo investigar são as características e preferências que contribuem para estabelecer contatos mais efetivos de empatia do aprendiz com o tutor.

O grupo de investigadores é interdisciplinar, partindo de referências prévias de psicologia (com base nas tipologias de personalidade de Jung; na teoria socionômica de J. L. Moreno, mais especificamente na sociometria; e a definição de uma teoria cognitiva/construtivista de aprendizagem, mais especificamente a Teoria de Aprendizagem Significativa de David Ausubel, bem como, motivação no processo ensino-aprendizagem; afetividade, emoções e sentimentos), Ergonomia (design dos agentes tutores) e Computação (Tutores Inteligentes e Computação Afetiva).

\section{Revisão de Literatura}

O Projeto: Um Estudo sobre Padrões de Perfis de Usuários e suas Preferências em Relação a Agentes Tutores define um SSPT (Sistema de Sugestão de Perfis de Tutores). Este estudo baseia-se nas escolhas mútuas (baseado nas preferências individuais) entre os agentes do sistema (humanos e virtuais) de acordo com a Teoria Sociométrica, de Moreno, e as tipologias de personalidade da Teoria Junguiana.

Para o SSPT existem 3 tipos de agentes o humano (usuário do sistema), o virtual (tutor) e o de perfis, sendo este último responsável pelo processamento e comparação dos perfis de tutor e de usuário.

Tanto os agentes humanos como os virtuais têm seu perfil armazenado no sistema na forma de um cadastro (definido por: gênero, área de conhecimento, idade e raça), e de um questionário com base na Tipologia de Personalidade segundo Jung.

A atividade dos tutores no sistema pode gerar melhores interações com o agente humano, potencializando a motivação do mesmo e conseqüentemente alterando o seu comportamento frente ao sistema. Com este cenário, são influenciadas também, a cognição e as emoções do usuário o que reforça mais ainda a sua motivação. 
Partimos do pressuposto que estas escolhas tem um caráter afetivo e expressam os desejos, sonhos, expectativas ou fantasias do usuário em relação ao tutor. Ou seja, a subjetividade do usuário estaria expressa através da sua escolha. E, mesmo no que se refere à vida cognitiva das pessoas não podemos, segundo Bock et alli (2002, p.190), deixar de lado a subjetividade e a afetividade, pois esta omissão implica numa visão parcial de homem.

Esta opinião não é unanimidade em todas as teorias psicológicas, mas está em conformidade com a visão de homem e as teorias que utilizamos para constituir esta pesquisa e que aparecem sistematizadas no Vê Epistemológico e nos mapas conceituais. Estas estratégias (Vê Epistemológico e Mapas) serão utilizadas durante todo o trabalho por serem importantes instrumentos de ensino e aprendizagem ${ }^{\mathrm{ii}}$.

\subsection{Afeto, Emoção e Sentimento}

Antigamente, até o século XIX, os termos: afeto, emoção e sentimento eram usados como sinônimos. Com o desenvolvimento da Psicologia, urge diferenciar-se as nuances que permeiam os mesmos. De acordo com Bock et al. (2002), (...) os afetos podem ser produzidos fora do indivíduo, isto é, a partir de um estímulo externo - do meio físico ou social(...), e refletem basicamente prazer ou desprazer. As emoções podem ser de: surpresa, raiva, nojo, medo, vergonha, tristeza, desprezo, alegria, paixão, atração física - algumas se apresentam, por vezes, de formas mais ou menos intensas, mais ou menos conscientes, estando também ligadas à sexualidade (amor).

Segundo Davidoff, (2004, p. 369). As principais emoções seriam: alegria, raiva, desagrado, medo, surpresa e tristeza, contudo, é possível incluir interesse, vergonha, desprezo e culpa, na mesma categoria de universalidade.

Os sentimentos, por sua vez, diferem das emoções pelo caráter menos intenso (menos "explosivo"), na forma de se manifestarem e por serem mais duradouros, não sendo acompanhado por manifestações orgânicas intensas.

\subsection{Gênero}

Optar pelo conceito de gênero significa alusão ao aspecto indefinido que envolve justamente a variável sexo, ou gênero nas abordagens teóricas, que por vezes não contemplam as diferenças exigidas pelos diversos modos de ser e fazer de homens e mulheres.

Não há consenso por parte da(o)s autora(e)s sobre a terminologia mais adequada, o que geralmente acontece quando se aborda conceitos que, para serem bem compreendidos, requerem uma abordagem interdisciplinar, já que envolvem várias áreas das Ciências Humanas.

Schienbinger (2001), considera importante diferenciar os termos "mulheres", "gênero", "fêmea", "feminino" e "feminista". De acordo com a autora:

"Uma "mulher" é um indivíduo específico; "gênero" denota relações de poder entre os sexos e refere-se tanto a homens quanto a mulheres; "fêmea" designa sexo biológico; "feminino" refere-se a maneirismos e comportamentos idealizados das mulheres num lugar e época específicos que podem também ser adotados por homens; e "feminista" define uma posição ou agenda política. (2001, p. 32”. 
A informação, que o sistema coleta, refere-se ao sexo dos usuários, que de forma descritiva aparece como homens (masculino) e/ou mulheres (feminino). Mas, no que tange à análise, a perspectiva terá por base a definição de Schienbinger, e os dados serão tratados conforme o conceito de gênero descrito na citação anterior.Tão importante quanto gênero temos de considerar raça.

\subsection{Raça}

Ao fazer o login no sistema o usuário também irá se autodefinir segundo os critérios censitários de cor/raça e esta é mais uma informação que será armazenada para a análise e possíveis cruzamentos. O mesmo critério é adotado na sugestão dos agentes tutores, cujas imagens foram selecionadas de acordo com as categorias definidas pelo Instituto Brasileiro de Geografia e Estatística (IBGE).

Machado (2004) faz referência aos dados obtidos pela PNAD 2002, p. 19, onde a variável cor/raça é classificada em cinco categorias:

"Branca, preta, amarela (compreendendo-se nesta categoria a pessoa que se declarou de raça amarela), parda (incluindo-se nesta categoria a pessoa que se declarou mulata, cabocla, cafuza, mameluca ou mestiça de preto com pessoa de outra cor ou raça) e indígena (considerando-se nesta categoria a pessoa que se declarou indígena ou índia). (PNAD - 2002, p. 19)”.

Vários autores, entre os quais Ricardo Henriques (2002), enfatizam a importância de articular gênero e raça, além de classe. Contudo, essa articulação está além dos objetivos deste trabalho, ficando limitada aos dois primeiros conceitos.

\subsection{Faixa etária}

Outro dado capturado pelo sistema é a idade do usuário. Além de constituir um elemento importante para analisar as diferenças de perfil, e inclusive permitir organizar os dados considerando as fases de desenvolvimento (por grupos de idade) em que o usuário possa se encontrar.

\subsection{Modelo de Motivação}

A motivação está presente em todas as atividades que desempenhamos em nossa vida, seja na escola, no trabalho ou no lazer. Neste trabalho, o objetivo é identificar elementos que favoreçam a motivação para a aprendizagem. Tal identificação constituise, em um dos grandes desafios da educação na atualidade, senão o maior deles. 


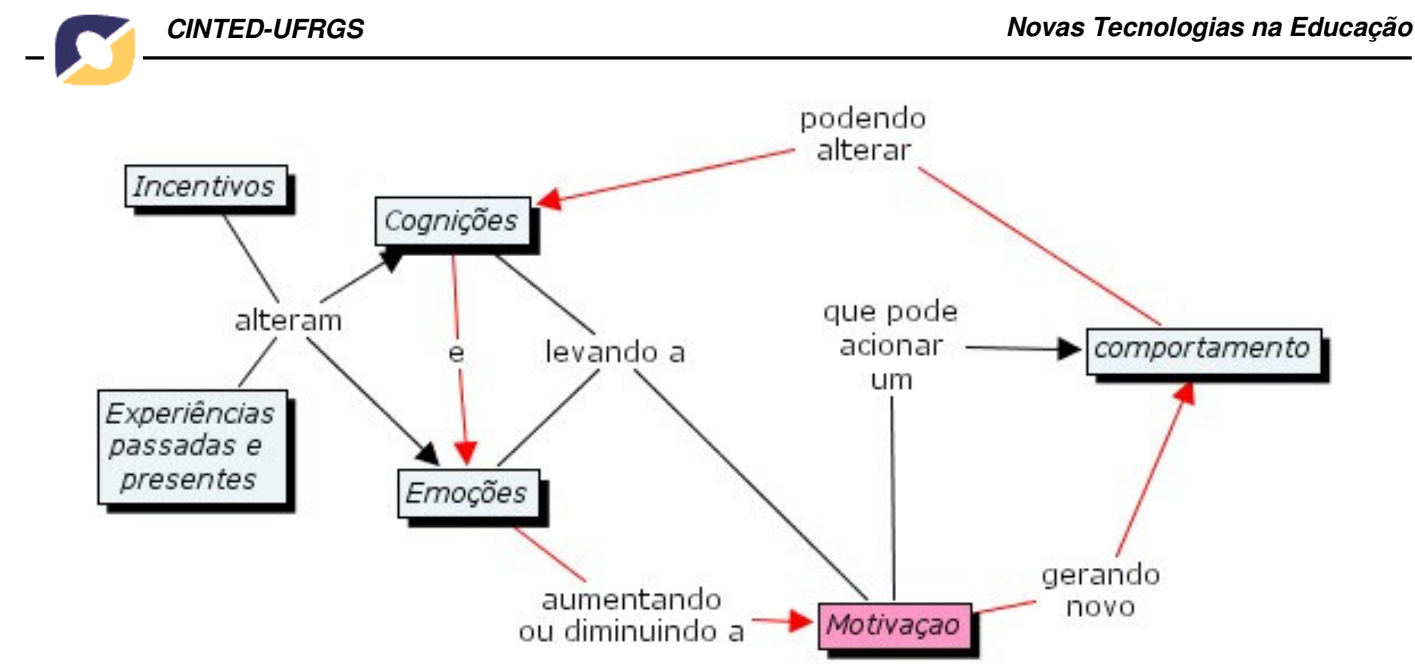

Figura 1: Mapa do modelo de motivação por incentivos ${ }^{\text {iii }}$

\subsection{Teoria Socionômica}

Jacob Levy Moreno (1993) desenvolveu a teoria socionômica a partir da década de 30 do século passado. Esta teoria, é utilizada até hoje e engloba a sociodinâmica, a sociometria e a sociatria. A parte da teoria que aborda as escolhas é a sociometria, é a partir dela, portanto, que se justifica a escolha do grupo e da temática. As escolhas podem ser: positivas, negativas ou indiferentes num determinado grupo, em relação a um critério específico.

As relações grupais para Moreno (idem, p. 45) baseiam-se no princípio do "Tele" ( do grego : distante, agindo à distância), que ele define como :

“(...) um ligação elementar que pode existir tanto entre indivíduos como, também entre indivíduos e objetos e que no homem progressivamente, desde o nascimento, desenvolve um sentido nas relações interpessoais (sociais). (...) e, pode assim, ser considerado como fundamento de todas as relações interpessoais (...) sadias. Repousa no sentimento $e$ conhecimento e conhecimento da situação "real*" das outras pessoas".

Sendo assim, faz-se necessário conhecermos os usuários e esses, por sua vez, têm o direito de "conhecer" e "escolher" o tutor que melhor lhe aprouver.

Para facilitar essa "escolha" e talvez possibilitar sugerir agentes tutores e estabelecendo um "padrão" de escolha com base no perfil do usuário, utilizaremos outra teoria, que não se contrapõe a essa, vindo a complementá-la. Essa teoria seria a de tipos psicológicos junguianos.

\subsection{Tipologia Junguiana}

A tipologia junguiana não é a única. Todas as tipologias representam,em linhas gerais, uma forma de identificar o que as pessoas têm em comum com as outras e também as suas diferenças.

As tipologias científicas podem ser divididas em três grandes grupos:

- Somática - se baseia na estrutura orgânica dos indivíduos;

- Somato-psíquica - se baseia na interação entre o orgânico e o psíquico;

- Psíquica - baseada somente em aspectos psicológicos. 
A tipologia psíquica oferece suporte teórico para trabalhar com elementos psíquicos e classifica os comportamentos humanos. Inclusa na tipologia psíquica está a tipologia Junguiana, que tenta definir estilos de comportamento individual e estilos cognitivos. Procura obter uma compreensão aproximada do fenômeno psíquico que é dinâmico. Esta tipologia considera a concepções de mundo e de homem que tem singularidade e conformidade.

O modelo tipológico de Jung (ou Tipologia Junguiana) é empiricamente observável, permitindo realizar testes, apesar de oferecer uma visão estática. Portanto, a análise dos testes que possam ser realizados deve ser feita considerando-se o dinamismo psíquico. Utilizamos, neste trabalho, um modelo que avalia a personalidade através das escolhas situacionais que cada usuário faz. Os resultados são fornecidos em um conjunto de três códigos que definem a atitude consciente e as funções mais e menos desenvolvidas (ou inconscientes).

Segundo padrão a descrição dos tipos pode ser:

a) Introvertidos

1. Sensação Introvertida com Pensamento Auxiliar

2. Pensamento Introvertido com Sensação Auxiliar

3. Intuição Introvertida com Pensamento Auxiliar

4. Sentimento Introvertido com Intuição Auxiliar

5. Pensamento Introvertido com Intuição Auxiliar

6. Sensação Introvertida com Sentimento Auxiliar

7. Sentimento Introvertido com Sensação Auxiliar

8. Intuição Introvertida com Sentimento Auxiliar

b) Extrovertidos

9. Sensação Extrovertida com Pensamento Auxiliar

10. Pensamento Extrovertido com Sensação Auxiliar

11. Sensação Extrovertida com Sentimento Auxiliar 12. Intuição Extrovertida com Sentimento Auxiliar 13. Intuição Extrovertida com Pensamento Auxiliar 14. Pensamento Extrovertido com Intuição Auxiliar 15. Sentimento Extrovertido com Sensação Auxiliar 16. Sentimento Extrovertido com Intuição Auxiliar

\subsection{Agentes}

No mundo físico, um agente é alguém que age em função dos interesses alheios, desempenhando papéis específicos (Ferreira 1994). Atualmente, sistemas baseados no paradigma de agentes têm recebido atenção especial, despertando o interesse por estudos mais aprofundados e sobre a possibilidade da utilização deste modelo para a construção de variadas aplicações, em diversas áreas da computação, embora não exista, ainda, uma definição precisa do que seja um agente nesse contexto (Hedberg 1995).

Uma das definições mais aceitas considera que um agente trata-se, normalmente, de um software atuando num papel corriqueiramente desempenhado por um usuário humano, podendo, ainda, agir em função de outro agente computacional (Johansen 1995), com o propósito de ajudar na realização de determinadas tarefas.

"É uma peça de software que executa uma determinada tarefa, empregando informações extraídas de seu ambiente para agir de forma adequada, no sentido de completar sua tarefa de modo bem sucedido. $O$ software deve ser capaz de adaptar-se a 
eventuais modificações ocorridas em seu ambiente de maneira que o resultado pretendido seja independentemente alcançado”.(Lecky-Thompson 1997) ".

"Chama-se de agente uma entidade abstrata que é capaz de agir sobre ela mesma e sobre seu próprio ambiente, que dispõe de uma representação parcial deste ambiente, e que, em um universo multiagente, pode comunicar-se com outros agentes, $e$ cujo comportamento é conseqüência de suas observações, de seu conhecimento e das interações com outros agentes". (Ferber 1991)".

Assim, percebe-se que para a realização de suas tarefas, surge a necessidade de especificar o comportamento destes agentes, bem como promover a comunicação entre eles, com os seus usuários e, também, com o ambiente em geral. Tais agentes podem realizar trabalhos rotineiros para os seus usuários, bem como auxiliá-los em tarefas complexas.

\subsubsection{Agente pedagógico}

Um agente pode ser considerado pedagógico, conforme Giraffa (1999), quando o sistema no qual está inserido baseia-se no paradigma de agentes e destina-se a propósitos educacionais. Um agente pedagógico atua no sentido de auxiliar no processo de ensino e de aprendizagem, observando as ações do usuário e interagindo com o mesmo, caracterizando-se como um tutor de conteúdos ou estratégias mais adaptadas ao perfil do usuário.

Algumas vezes, o agente pedagógico pode assumir a roupagem de um personagem que dialoga explicitamente com o usuário. Além de atuar como tutor, com estratégias e conteúdos adaptados ao perfil do usuário, o agente pedagógico pode agir, se assim for concebido, como companheiro virtual de aprendizagem, com o papel de motivar o usuário.

\subsubsection{Agente companheiro virtual de aprendizagem}

O conceito de agente companheiro virtual de aprendizagem (learning virtual companion), proposto por Picard (2004), abarca a questão da afetividade, definindo tal agente como um personagem afetivo que acompanha o usuário nos seus estudos e na realização de suas tarefas, buscando estabelecer relações de empatia e mantê-lo motivado a persistir apesar das dificuldades no processo.

Ao contrário da maioria dos Sistemas Tutores Inteligentes - e de agentes pedagógicos concebidos apenas como tutores -, que trazem uma abordagem voltada à manipulação de tarefas (níveis de dificuldade e focos de interesse), a proposta dos agentes companheiros virtuais de aprendizagem é uma abordagem afetiva, que permita ao usuário a visualização de que a dificuldade faz parte do processo, de que algumas tarefas são mais difíceis do que se gostaria e não podem ser decompostas ou transpostas, por isso é preciso estar preparado para enfrentá-las. Um agente companheiro de aprendizagem estabelece vínculo e pretende comprometer, responsabilizar, o usuário com o seu próprio processo de aprendizagem. 


\section{Metodologia e definição do sistema}

A metodologia utilizada no presente trabalho pode ser descrita em três partes:

1) Inicialmente utilizamos estratégias metodológicas da Teoria Cognitiva/Construtivista de David Ausubel e D. B. Gowin, sobre mapas conceituais e o vê epistemológico.

2) Posterior a esta etapa, quando já havíamos elaborado dois mapas e definido a "rota" ou "proposição-chave" que constituiria o eixo da pesquisa, foi criada, também de forma cooperativa, a definição do sistema.

3) A última etapa a ser definida, em termos metodológicos, está inserida na definição do sistema, mas precisa de maior detalhamento. Esta seria a parte de análises estatísticas dos dados coletados.

Como produto das teorias compiladas neste trabalho surgiu a idéia de implementação de um protótipo do Sistema de Sugestão de Perfis de Tutores (SSPT). Este serviço teria como principais funcionalidades:

- A classificação dos usuários do sistema conforme a Tipologia de Personalidade Junguiana (Jung, 1967, p.386-552);

- A sugestão de perfis de tutores que mais se assemelham ao perfil psicológico do usuário (op.cit.).

Essas informações obtidas pelo questionário com base na Tipologia de Personalidade seriam combinadas a dados pessoais do usuário tais como gênero, raça, sexo, idade e área profissional. Com base nesta combinação imagina-se estimar alguma relação estatística entre a quantidade de escolhas sobre um determinado perfil de tutor e os perfis dos usuários que os escolheram.

Após a coleta das informações do usuário, que o sistema seja capaz de sugerir um tutor (dentre os definidos em suas bases de dados) com características similares às do usuário do sistema (as informações obtidas aqui servirão como base para a validação das hipóteses levantadas no trabalho).

\section{Escolha das imagens dos tutores sugeridas pelo sistema}

As fotos dos tutores apresentadas ao usuário foram escolhidas com vistas a manter um padrão quanto a características como: faixa etária, definida em torno de 40 anos; cor do cabelo, indicativa da raça e evitando extremos; e ainda, características como uma aparência agradável e um perfil simpático. Por meio da homogeneidade de tais características buscamos atenuar quaisquer escolhas que interferissem na finalidade de definir a preferência do usuário quanto à raça e sexo de seu tutor.

As fotos foram obtidas através de bancos de dados de imagens na Web, e utilizadas neste primeiro momento do estudo, de forma representativa, visto que os direitos autorais ainda não foram devidamente efetivados.

\section{Considerações finais}

Estamos acostumados com estruturas que tem princípio, meio e fim. Até como uma analogia à própria vida. Sendo assim, não poderíamos deixar de fazer algumas considerações finais sobre o estudo, a título de fechamento.

O sistema foi concebido, inicialmente, com o objetivo de potencializar a aprendizagem baseada na aceitação mútua por parte do aprendiz e do tutor. Este último servindo como agente motivador no processo de aprendizagem. 
Apesar de termos concluído o protótipo do Sistema de Sugestão de Perfis de Tutores (SSPT), este ainda não foi testado, a não ser nas suas funcionalidades básicas. Sendo assim, não existem, no momento elementos que nos permitam emitir qualquer tipo de conclusão sobre a validade, fidedignidade e efetiva possibilidade de utilização do SSPT.

O que podemos, por hora, é extrapolar no que tange a possibilidades de utilização do sistema:

1) Se o SSPT for aprovado para sugestão de agentes tutores, a análise estatística permite que, com um nível de confiança satisfatório (entre 95\% e 99\%) e com ajustes de contexto, possamos elaborar protótipos e sugerir agentes tutores para uso na Universidade Federal do Rio Grande do Sul (UFRGS) e outras instituições de ensino.

2) Além de atingir o objetivo, é possível, que durante a realização do estudo ocorram alguns desdobramentos, tais como: o desenvolvimento de protótipos adequados às necessidades específicas de padrões de usuários, segundo: as áreas de conhecimento, gênero, raça e/ou grupos de idade.

Isso implicaria na manutenção de uma linha de pesquisa em caráter permanente no Programa de Pós-Graduação em Informática na Educação da UFRGS.

\section{Referências bibliográficas}

BOCK, Ana Mercês Bahia; FURTADO, Odair; TEIXEIRA, Maria de Lourdes Trassi. Psicologias: uma introdução ao estudo de psicologia. —13 ed. reform. e ampl. — São Paulo: Saraiva, 2002.

BOGDAN, Robert; BIKLEN, Sari. Investigação qualitativa em educação: uma introdução à teoria e aos métodos. Tradução: Maria João Alvarez, Sara Bahia dos Santos e Telmo Mourinho Baptista. Portugal: Porto editora, 1994.

DAVIDOFF, Linda L. Introdução à psicologia. Tradução: Lenke Perez. 3 ed. São Paulo: Makron Books, 2001.

ETZIONI, Oren; WELD, Daniel S. Intelligent Agents on the Internet: Fact, Fiction, ant Forecast. IEEE Expert, New York, p. 44-49, Aug. 1995.

FERBER, Jacques; GASSER, Les. Intelligence Artificiclle Distribuée. In: Conference on Expert Systems and Their Applications, 11., 1991, Avignon. Tutorial Notes... [S.l.:s.n.], 1991.

FROZZA, Rejane et ali. Artigo disponível em http://www.inf.ufes.br/ sbie2001/figuras/artigos/a041/a041.htm

GIRAFFA, Lúcia M. M. Uma arquitetura de tutor utilizando estados mentais. 1999. Tese (Doutorado em Ciências da Computação) - Instituto de Informática, UFRGS, Porto Alegre.

HARTSON, H. Rex. Human-computer interaction: Interdisciplinary roots and trends. Elsevier Science Inc., The Journal of Systems and Software, Department of Computer Science, Virginia, USA, 43, p. 103-118, 1998.

HEDBERG, Sara Reese. Intelligent Agents: The First Harvest of Softbots Looks Promising. IEEE Expert, New York, p. 6-9, Aug. 1995.

HENRIQUES, Ricardo. Raça e gênero no sistema de ensino: os limites das políticas universalistas na educação. Brasília: UNESCO, 2002.

JAQUES, Patrícia A. Using an Animated Pedagogical Agent to Interact Affectively with the Student. 2004. Tese (Doutorado em Ciências da Computação) - Instituto de Informática, UFRGS, Porto Alegre. 
JOHANSEN, Dag et al. An Introduction to the TACOMA Distributed System - Version 1.0. 1995. Disponível por WWW em http://www.cs.uit.no/DOS/Tacoma/ (12 de dezembro de 2004).

JOHNSON, Lewis; RICKEL, Jeff; LESTER, James. Animated Pedagogical Agents: Face-to-Face Interaction in Interactive Learning Environments. International Journal of Artificial Intelligence in Education, 11, pp. 47-78. Disponível na WWW em http://www.csc.ncsu.edu/eos/users/l/lester/www/imedia/apa-ijaied2000.html acessado em novembro de 2001.

JUNG, Carl Gustav. Tipos psicológicos. Rio de Janeiro: Zahar, 1967.

LECKY-THOMPSON, J. W. Software Agents Mail List. 1997. Disponível por WWW em http://www.ee.mcgill.ca/ agent_faq.html (30 de julho de 1997).

MACHADO, Nádie Christina Ferreira. Letramento, gênero, raça e ocupação no Brasil. Dissertação de mestrado, no Programa de Pós-Graduação em Educação da UFRGS, Porto Alegre, 2004. Carvalho, Marie Jane Soares, orient. 124p.

MOREIRA, Marco Antonio; BUCHWEITZ, Bernardo. Novas estratégias de ensino e aprendizagem: os mapas conceituais e o vê epistemológico. Lisboa: Plátano, 1993.

MORENO, Jacob Levy. Psicoterapia de grupo e psicodrama. Tradução: Dr. Antônio C. Mazzaroto Cesarino Filho. Campinas/SP: Editorial Psy, 1993.

PAIVA, A.; MACHADO, I. Vincent, na autonomous pedagogical agent for on-thejob training. In: Proceedings of Intelligent Tutor Systems Conference, SpringerVerlag, 1998.

PORTO, Paulo Ricardo Prestes; PALAZZO, Luiz Antonio Moro; CASTILHOS, José Mauro Volkmer de. Agentes de Informação Inteligentes. In: Oficina de Inteligência Artificial, Pelotas, 1., 1997. Anais... Pelotas: Ed. EDUCAT, 1997. p. 81-107.

SCHIENBINGER, Londa. O feminismo mudou a ciência? Tradução de Raul Fiker. Bauru, SP: EDUSC, 2001.

TRIOLA, Mario F. Introdução à estatística. Tradução: Alfredo Alves de Farias. 7 ed. Rio de Janeiro: LTC, 1999.

WOOLDRIDGE, M.; JENNINGS, N. Agents Theory, Architectures and Languages: A Survey. In: WOOLDRIDGE, M.; JENNINGS, N. (Eds.).

\footnotetext{
${ }^{1}$ Nota dos autores: entenda-se onde utilizamos o termo "Tutor", ao longo do texto, estamos fazendo referência ao conceito de "Agente Companheiro de Aprendizagem". Conforme aparece definido na seção 2.8.2.

${ }^{2}$ Além de terem outros usos, como ferramentas de avaliação e análise de currículo, independente da área de conhecimento. Os mapas decorrem da Teoria da Aprendizagem Significativa de David Ausubel, e foram elaborados na versão 3.6 do CMap Tools (ferramenta elaborada pela University of West Flórida); o Vê epistemológico constitui-se em instrumento heurístico, cuja finalidade é auxiliar professores e alunos a perceber quais os elementos envolvidos na produção de conhecimento. Ambos são estratégias metacognitivas, incluindo meta-aprendizagem. (Moreira, 1993)

${ }^{3}$ Fonte: Elaborado a partir de esquema proposto por Davidoff (2001, p. 327). O mapa representa, de forma cíclica (ou sistêmica) como a partir de incentivos é possível alterar o nível motivacional dos usuários promovendo novos comportamentos. No caso, o comportamento desejado está relacionado diretamente aos aspectos cognitivos e emocionais do processo de ensino e aprendizagem mediada por computador.
} 